\title{
Photometric Effects of Accretion Disks in Long-Period Eclipsing Binaries
}

\author{
Edward C. Olson \\ Astronomy Department \\ University of Illinois \\ $1011 \mathrm{~W}$. Springfield Ave. \\ Urbana, Illinois 61801 USA
}

(Received 20 October, 1988)

\section{INTRODUCTION}

Accretion disks are invoked in a variety of astrophysical settings, ranging from stellar-mass black holes to active galactic nuclei. There is now little doubt that true accretion disks can also occur in binaries containing non-degenerate stars (Peters 1980; Plavec et al. 1984; Polidan 1987). In this paper, I will discuss some of the properties of disks observed photometrically in the long-period systems $\mathrm{KU}$ Cyg and RZ Oph.

The most intensively-studied disks are of course those in cataclysmic binaries. Accretion disks in such binaries are thought to have the classical 'alpha' structure of Shakura and Sunyaev (1973), in which viscous dissipation arising from differential rotation converts gravitational potential energy to thermal energy in supplying the disk luminosity. This physical process requires that the viscous time scale be comparable to the radiative decay time of the disk (Pringle 1981). Most of the disk's volume is optically thick, so disk luminosity is estimated by integrating black-body or stellar atmospheric fluxes over the disk (see Kriz and Hubeny 1986 for qualifications).

For several reasons, large accretion disks that may form in non-degenerate binary systems cannot have this classical structure. First, the radiative decay time for a large disk, such as the one in RZ Oph (O1son 1987), is much shorter than the viscous decay time; some other mechanism provides dissipation. Second, large disks cannot be optically thick normal to the orbital plane, because such disks would radiate something like a low-gravity stellar atmosphere, and would require an enormous energy source. As described below, disks in $\mathrm{RZ}$ Oph and $\mathrm{KU}$ Cyg (O1son 1988) are optically thin in the vertical direction; they are cool enough that their extinctions are low, and contain essentially no thermal neutral hydrogen contribution.

\section{EXPECTED PHOTOMETRIC EFFECTS OF ACCRETION DISKS}

In a cataclysmic binary, the large potential well down which matter falls to form the disk results in a relatively luminous, easily-detected, disk. In non-degenerate binaries, disk luminosities are comparable to, or less than, that of the component stars. While non-degenerate binaries are often much brighter than cataclysmics, to reveal the physical nature of their disks requires long-term photometry with a precision on the millimagnitude level, extending from the ultraviolet to the near infrared. Only a few observatories are willing or able to support this kind of observational program. 
In the binaries we consider, the hotter star ('gainer') is circled by an accretion disk, whose matter is supplied by a cool loser. The loser may or may not fully fill its critical Roche lobe. If a luminous disk exists in such an eclipsing system, we expect two distinct signatures in the light curves: (1) before and after primary stellar eclipse, the cool star partially eclipses the disk, producing 'dips' bordering the stellar eclipse (see e.g. Hall, Cannon, and Rhombs, 1984, and Stuhlinger, Shaw, and Hall, 1984, for examples in RS Cep); (2) around secondary eclipse, the disk itself partially occults the cool star (Shao, 1967, and Koch, 1972, noted this effect in SX Cas). Both effects are clearly present in $\mathrm{RZ} O \mathrm{Oph}$ and $\mathrm{KU} \mathrm{Cyg}$, as shown below. If these effects can be observed with sufficient precision, then both the emitting and the purely transmitting properties of disks can be deduced. As it turns out, both are essential to a modest understanding of these disks. If a disk is larger than the cool star, then what might have been a total primary eclipse looks partial, because disk light is always present.

It should also be noted that disks affect spectra. A good example is Popper's (1964) observation of $\mathrm{KU}$ Cyg in totality. He noted a contaminating continuum, appearing near 4000A and increasing toward the 3500A observing limit, and not arising from Balmer emission. The contaminating light in fact is from the accretion disk (Olson 1988). Other spectroscopic effects should also be produced by such disks.

A11 of the observations used in this paper were made at the Mount Laguna Observatory on the Ströngren-Crawford uvby system, to which a near-infrared intermediate-band filter close to the Kron $I$ was added. All data are corrected for differential extinction and standardized. Comparison and check stars were observed regularly. Further observational details can be found in olson and Stoehr (1986) and Olson (1987).

\section{KU CYGNI}

$\mathrm{KU}$ Cyg (BD $+46^{\circ} 2879 ; \mathrm{F} 4 \mathrm{p}+\mathrm{K} 5 \mathrm{III} ; \mathrm{P}=38.4$ days) was studied extensively by Popper (1964, 1965), both spectroscopically and photometrically. My five years' photometry of this binary shows, in agreement with Popper, that brightness variations are significant and confusing. Yellow observations obtained from 1982 to 1987 are shown in Fig. 1. Clearly, wholesale vertical shifts in light levels occurred during this time. In addition, night-to-night fluctuations are present between phases 0.5 and 1.0 . Such variations increase toward short wavelength. The slow component can often be effectively removed with judicious wholesale brightness shifts (01son 1988). The resulting adjusted infrared light curve, shown in Fig. 2, is relatively clean, if abnormal. Popper has shown that the orbit is probably circular; therefore, the observed secondary eclipse is abnormally wide. Stellar primary eclipses are we11-defined, and yield reasonably consistent fractional stellar radij and orbital inclination. The dashed curve of Fig. 2 then shows the predicted light variation outside primary eclipse, synthesized with the Wilson-Devinney code under the naive assumption that only stellar light is present. Both diskproduced effects are clearly present, and effect (2) has notably broadened and deepened secondary eclipse. The latter distortion decreases toward short wavelength, and disappears in the ultraviolet. (See Fig. 3 for blue observations). Following procedures used with R2 Oph (Olson 1987), the ratio of disk to cool-star light in primary eclipse totality was derived, and is in 
complete agreement with Popper's spectroscopic results, noted earlier. That is, non-stellar light dominates the cool-star light in totality shortward of about $4000 \mathrm{~A}$.

Photometric signature 1, abnormal dips bordering primary eclipse, are clearly evident in Fig. 2. Because light curves are particularly unstable between secondary and primary eclipse, only the dip following eclipse was used to determine the disk emission. The relative monochromatic flux lost in the dip at external eclipse contact, relative to the yellow flux of the hot star, is shown in Fig. 4. This distribution is corrected for a reddening of $\mathrm{E}_{\mathrm{by}}=$ 0.15 , and the observational error spread is shown.

The most striking result in KU Cyg pertains to the disk transmission and the shape of secondary eclipse. I first calculated the occulting effect on the cool-star light of a disk with gravitational stratification, gaseous-phase extinction, temperature, and pressure which gives an emitted spectrum in agreement with the observed disk emission. Such a disk fails, by nearly an order of magnitude, to provide adequate light blocking to match the observed secondary eclipse. To reach agreement with observations, we must: (1) increase the disk scale height by about three times; (2) add an extinction source which has no re-emission in the optical spectrum. We could speculate on several levitating mechanisms to thicken the disk (the observation made first by $R$. Crawford (1981) of supersonic emission line widths in circumstellar matter in $U$ Cep may be relevant to this issue). The extra extinction would naturally seem to be dust acquired from the outer atmosphere of the cool loser. I refer to this disk as 'thick and somewhat dusty'.

The dust, which provides most of the blocking of the cool star's light in secondary eclipse, is not spread through the disk in proportion to the gas pressure. Dust is probably absent close to the hot star; in this model, it was arbitrarily confined to the outer half of the disk. Moreover, dust apparently evaporates completely in the relatively dense regions close to the orbital plane. Otherwise, neither much of the disk emission nor most of the hot star's light could escape from the disk. In one successful disk model, the radial distribution of temperature runs from about $5300 \mathrm{~K}$ to $4600 \mathrm{~K}$, and gas pressure $\log P$ drops from about 2.3 to 1.2 . Under these conditions, the major gaseousphase extinction comes from Rayleigh scattering from neutral hydrogen, $\mathrm{H}^{-}$ absorption, and electron scattering. The dust/gas mass ratio is about $10^{-6}, a$ value well below that in the interstellar medium (though possibly

characteristic of the outer layers of the cool loser). However contrived the dust distribution seems to be, it is the only distribution that yields agreement with all observations of $\mathrm{KU}$ Cyg. Results of this model are shown as open circles in Figs. 2 and 3, and as diamonds in Fig. 4. The disk emission is very red, and non-stellar.

In connection with 'thick disks', it is relevant that Plavec (1988) had to invoke a thick, non-alpha, disk in his model of TT Hyd. There is a hint that the disk in RZ Oph may be slightly 'thick and dusty'. Shaviv and Wehrse (1986) suggest that disk thicknesses have been underestimated in classical alpha disks.

We finally note that the slow brightness variations in $\mathrm{KU}$ Cyg are not present in primary eclipse totality, so the cool star cannot be their source (in agreement with Popper's conclusion). Monochromatic flux variations suggest 
that the hot star is responsible, and that the bright 'high' state results from elevated mass accretion by the gainer. The luminosity difference between high and low states is about one-third of the low-state luminosity. The peak incremental accretion rate onto a synchronously rotating gainer is $\approx 4 \times 10^{-7}$ solar masses per year. If the hot star rotates super-synchronously, as seems likely, then the rate is correspondingly higher.

\section{RZ OPHIUCHI}

Both accretion disk signatures are present in RZ Oph (BD $+07^{\circ} 3832$; F5Ib + K5Ib; P - 262 days), as shown in Fig.5. The dashed line is a light curve synthesized with the Wilson-Devinney program assuming only stellar light, and using the radii and inclination of Knee et al. (1986). Anomalies due to an accretion disk around the hotter star are generally similar to those in $\mathrm{KU}$ Cyg. There are, however, two obvious asymmetries: first, the dip following primary eclipse is largely missing, and second, secondary eclipse is asymetrical. Note that the dip before primary eclipse and secondary eclipse ingress both involve the leading half of the disk. The dip begins with the ecli: se of the disk by the cool star near phase 0.8 , while secondary eclipse starts near phase 0.4 , as the disk begins to eclipse the cool star. Therefore, the processes responsible for disk emission and for light-blocking have different radial distributions within the disk. As in $\mathrm{KU} \mathrm{Cyg}$, most of secondary eclipse is due to light scattering by dust in the disk. Indeed, that is manifestly the case, since the following half of the disk produces little emission, but leads to an extended secondary eclipse egress. The only caveat here is a warning that different phase ranges have of necessity been observed in different orbital cycles. Cycle-to-cycle changes could complicate the interpretation.

Analysis of the pre-eclipse dip suggests that the disk temperature runs radially from about $5600 \mathrm{~K}$ to $4400 \mathrm{~K}$. Fig. 6 shows the observed monochromatic flux distribution of the light lost near first contact, relative to the yellow flux of the hot star. Diamonds show model results. As in the case of $\mathrm{KU} C \mathrm{CYg}$, the disk light is very red, reflecting the increase of scattering coefficients toward short wavelength.

Test calculations suggest that the dip following primary eclipse will be nearly invisible if the mean disk temperature falls to about $4000 \mathrm{~K}$. The leading half of the disk is therefore at least $1000 \mathrm{~K}$ hotter than the following half. Since the cool star fails to fill its Roche lobe (Olson and Hickey 1983; Knee et al. 1986), it is likely that the disk forms from a stellar wind. That wind is probably considerably amplified over the rate from a single star (Kenyon 1988). The hot gainer is carried by the relative stellar orbital motion into this wind at about $30 \mathrm{~km} / \mathrm{sec}$, a supersonic rate. It is possible that shock heating could account for the temperature difference between the halves of the disk. I estimate the shock-induced luminosity to be of order $5 \times 10^{31} \mathrm{erg} / \mathrm{sec}$, compared to the kinetic energy of the hot star in the centerof-mass frame of about $2.4 \times 10^{45} \mathrm{erg}$. Thus, dissipation of the hot star's energy would produce significant orbital decay in about $3 \times 10^{6}$ years. For as long as the wind lasts, this dissipation will influence the orbital evolution of the binary system, and the binary will behave somewhat as a common-envelope system.

If such dissipation is actually occurring in $R Z$ oph, then the orbital period should be decreasing. Baldwin (1978) analyzed all available times of 
minima, and concluded that there is no evidence for a period change. Since his work, several photoelectric times of minima have become available. These new minima have $(0-C)$ 's of about -0.2 day on Baldwin's ephemeris, so I decided to look again at this question. In referring to the original sources of early visual timings, a few misprints were found and corrected. Before cycle count 54 in Baldwin's ephemeris, there are 28 visual times; I averaged these into four normals with seven observations each. Two minima (E--36 and -29 ) were combined into a single normal, and photoelectric minima were added. A weighted least-squares solution gives:

$$
\text { Min } I(H J D)=2,442,204.221+261.92623 \mathrm{E}
$$

(O-C)'s from this emphemeris are shown in Fig.7, with estimated mean errors for the visual normals. One certainly cannot conclude from these data that the period is changing. Perhaps not surprisingly, a parabolic fit to these observations reduces the predicted mean error per point by about $20 \%$, relative to the linear ephemeris. This fit implies a period change $\Delta P / P \approx-2 \times 10^{-7}$ which is at least of the correct sign for the proposed effect. Unfortunately, many years will pass before proof of a period decrease and its magnitude can be found.

If this view of shock dissipation in the leading half of the disk is correct, then it is not surprising that emission and light-blocking have different radial distributions in the disk. Dust may evaporate completely in shocked regions. Considerable dust may also collect and survive in the following half of the disk. It remains to model the egress half of secondary eclipse, which is produced by the following half of the disk. The 'levitating effect' necessary in $\mathrm{KU}$ Cyg is not needed in $\mathrm{RZ} O \mathrm{ph}$, nor is it necessary to clear the central-plane regions of dust (though the model does not preclude such a clearing). The shape of the light curve depends mainly on the assumed radial distribution of pressure (or density) in the disk. This distribution in turn fixes the dust-scattering cross section per unit volume (where present, I assume that the dust-scattering coefficient per gram is constant). The central-plane gas pressure is assumed to be:

$$
P(r)=P_{0} \exp \left(-A r / R_{D}\right) \text {, where }
$$

$R_{D}$ is the outer radius of the disk, and $A$ is determined empirically for this disk to be about 6 . The resulting predictions are shown as open circles in Fig. 5. The dust-to-gas ratio is about $0.5 \times 10^{-7}$, so this disk is less contaminated by dust than is the disk in KU Cyg. This model at least demonstrates that it is possible to reach reasonable agreement with observations .

\section{NEW OBSERVATIONS OF BOTH BINARIES}

It would obviously be desirable to observe both pre- and post-primary eclipse dips in $R Z$ Oph in the sane orbital cycle. It will be possible to do so during 1989, and we intend to observe RZ Oph photometrically and spectrographically throughout the roughly 100-day interval. Another longperiod system whose light curves are similar to those of KU Cyg is RX Cas (Kriz et al. 1980). Since the cool loser in this system is a gG3, an accretion disk 
around the hot star should be essentially free of dust. We will attempt to obtain five-color photometry of this system to test this expectation. The presence of accretion disks and their effects on binary star light should also be tested spectroscopically. In particular, resonance absorption lines at long wavelengths, such as the Na D-lines, should vary in strength as the disk eclipses the cool star. Their behaviour should provide further constraints on the structure of the disk. A search for infrared calcium triplet emission may also prove to be fruitful (e.g., Persson 1988).

\section{SUMMARY}

A multi-year photometric program on long-period eclipsing binaries has begun to uncover some properties of accretion disks in these systems. Emission and transmission properties can sometimes be found from light curve features produced by partial eclipses of the disk by the cool star, and by partial occultations of the cool star by the disk. These disks do not have the classical alpha structure. They are optically thin normal to the orbital plane, but may be geometrically thicker than purely gravitationally-stratified disks. Disk gas may be contaminated by dust particles acquired from the outer layers of the cool loser. In some systems, 'high' states, produced by elevated mass accretion by the hot star, occur, suggesting that the mass distribution in the disk is clumpy. However mass-transfer rates are found, they lie between $10^{-7}$ and $10^{-6}$ solar masses per year.

While this binary sample is small at the moment, some of its properties are shared with other systems. The author has five-color observations of about a dozen additional systems, which may fill out this picture more fully.

\section{ACKNOWLEDGEMENTS}

The author thanks the U. S. National Science Foundation for support of this work. The Research Board of the University of Illinois provided about half of the computing funds. The director and staff of Mount Laguna Observatory provided excellent technical support and much assistance with observations, which required years of flexible scheduling to cover light curve events. The author thanks the faculty and staff of the UCLA Astronomy Department, and especially Drs. Mirek Plavec and Dan Popper, for hospitality and much assistance during a sabbatical leave when a first draft of this paper was written. Much helpful advice was provided as well by Drs. Dick Crawford, Paul Etzel, Mario Livio, Norm Markworth, Gerry Peters, Ron Polidan, Jim Pringle, Joe Smak, Brian Warner, Ron Webbink, Bob Wilson, and Art Young. San Diego State University observers were Clayton Heller, Jeff Hickey, Linda Humes, Karen Kramer, Vickie Paylor, Tom Santrizos, Juan Tamez, Scott Temple, and Mark Whittaker. The Mount Laguna observatory is operated jointly by San Diego State University and the University of Illinois in Urbana/Champaign.

\section{REFERENCES}

Baldwin, B. W. (1978). Astrophys. J. 226, 937.

Crawford, R. C. (1981). Thesis, UCLA Astronomy Department.

Hall, D. S., Cannon III, R. O., and Rhombs, G. G. (1973). Publ.

Astron. Soc. Pac. $\underline{85}, 420$.

Hall, D. S., Cannon III, R. O., and Rhombs, C. G. (1984). Astron. 
J. 89,559 .

Hall, D. S., and Stuhlinger, T. (1978). Astron. Acta 28, 207. Hal1, D. S., and Walter, K. (1975). Astron. Astrophys. 38, 225. Kenyon, S. J. (1988). Astron. J. 96, 337.

Knee, L. B. G., Scarfe, C. D., Mayor, M., Baldwin, B. W., and Meatheringham, S. J. (1986). Astron. Astrophys. 168, 72.

Koch, R. H. (1972). Astron. J. 71, 500 .

Kriz, S., and Hubeny, I. (1986). Bull. Astron. Inst. Gzechosl. 37,129 .

Kriz, Arsenijevic, J. Grygar, J., Harmanec, P., Horn, J., Koubsky, S., Pavlovski, K., Zverko, J., and Zdarsky, F. (1980). Bul1. Astron. Inst. Czechos1. 31, 284.

Olson, E. C. (1987). Astron. J. 94, 1309 .

Olson, E. G. (1988). Submitted to Astron. J.

Olson, E. C., and Hickey, J. P. (1983). Astrophys. J. 264, 251.

Olson, E. C., and Stoehr, C. A. (1986). Astron. J. 91, 1418 . Persson, S. E. (1988). Publ. Astron. Soc. Pacific $100,710$. Peters, G. J. (1980), in Close Binary Stars : Observation and Interpretation, ed. M. J. Plavec, D. M. Popper, and R. K. U1rich, p 287.

Plavec, M. (1988). Astron. J. 96, 755.

Plavec, M., Dobias, J. J., Etzel, P. B., and Weiland, J. E. (1984), in Future of Ultraviolet Astronomy Based on six Years of IUE Research, p 240 .

Polidan, R. S. (1987). Bu11. Amer. Astron. Soc. 19, 709.

Popper, D. M. (1964). Astrophys. J. 139, 143.

Popper, D. M. (1965). Astrophys. J. 141, 314.

Pringle, J. E. (1981). Annu. Rev. Astron. Astrophys.19, 137.

Shakura, N. I., and Sunyaev, R. A. (1973). Astron. Astrophys. 24 , 337.

Shao, C-Y (1967). Astron. J.72, 480.

Shaviv, G., and Wehrse, R. (1986). Astron. Astrophys. 159, I5.

Stuhlinger, T., Shaw, J. S., and Hall, D. S. (1984). Astron. J. $\underline{89}, 562$.

Wilson, R. E., and Plavec, M. (1988). Astron. J. 95, 1828. 


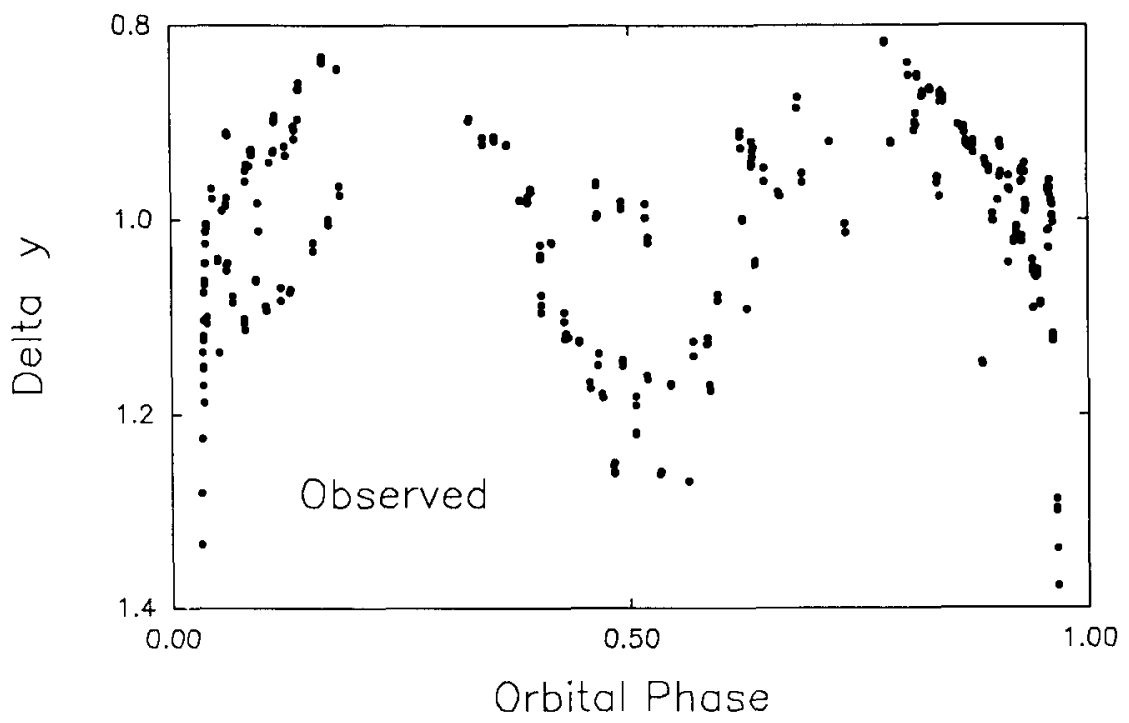

Fig. 1. Yellow observations of $\mathrm{KU}$ Cyg, mostly outside primary eclipse, obtained from 1982 to 1987. Four-point running normals are plotted. Most of the observed scatter before phase 0.5 is due to a slow, quasicyclical brightness variation of the entire system, similar to the variation seen in $\mathrm{RX}$ Cas. 


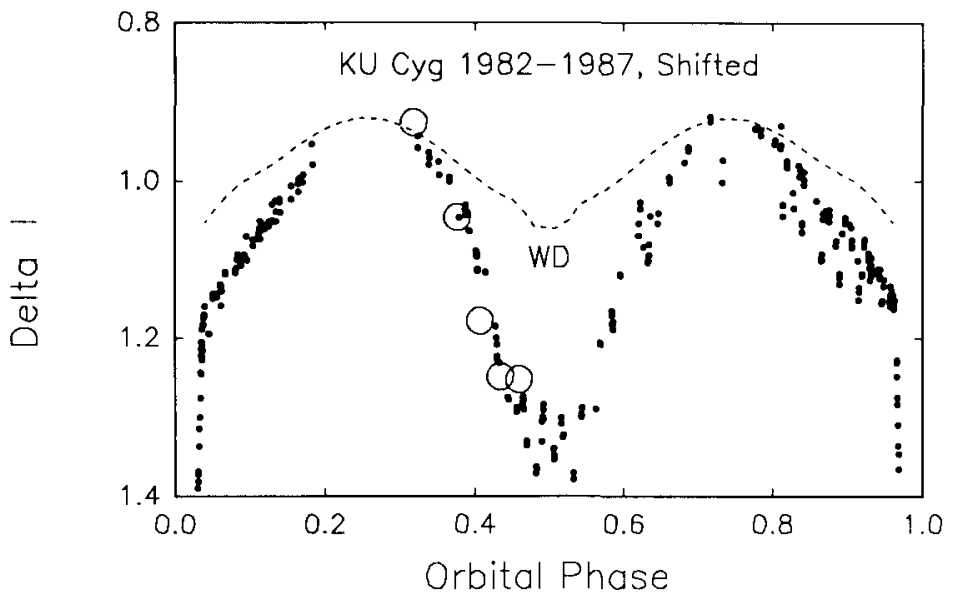

Fig. 2. Infrared observations of $\mathrm{KU} \mathrm{Cyg}$, with the slow variation shifted out. Significant scatter remains between phases 0.5 and 1.0 , where the observer sees the region where stream and disk interact. These variations increase in size toward short wavelength. Open circles are predictions of a thick, dusty disk model (see text).

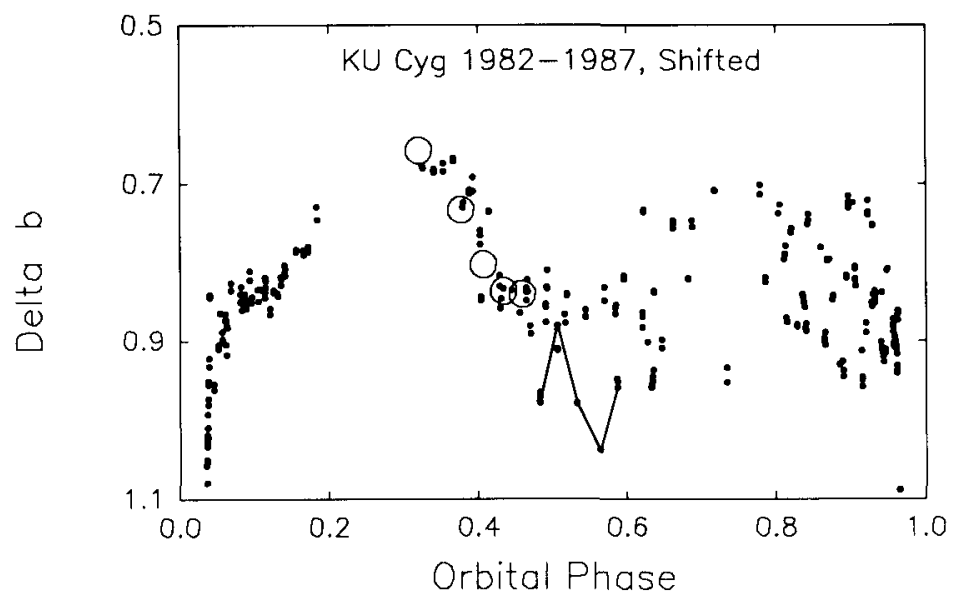

Fig. 3. Shifted blue observations of $\mathrm{KU}$ Cyg. Secondary eclipse is shallower than it is at longer wavelengths. Straight lines connect normals obtained on five consecutive nights, and clearly show the nightly variations that are confined mainly to phases between 0.5 and 1.0 . 


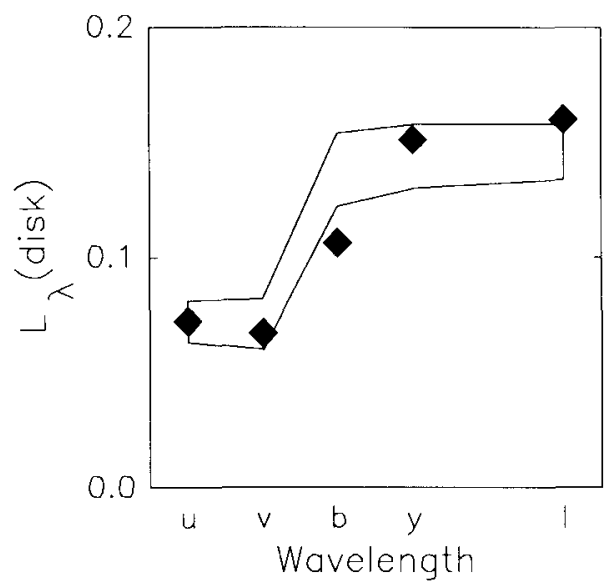

Fig. 4. The monochromatic flux distribution of disk 1ight lost just after fourth contact of primary eclipse. Diamonds are predictions of a thick, dusty disk model (see text).

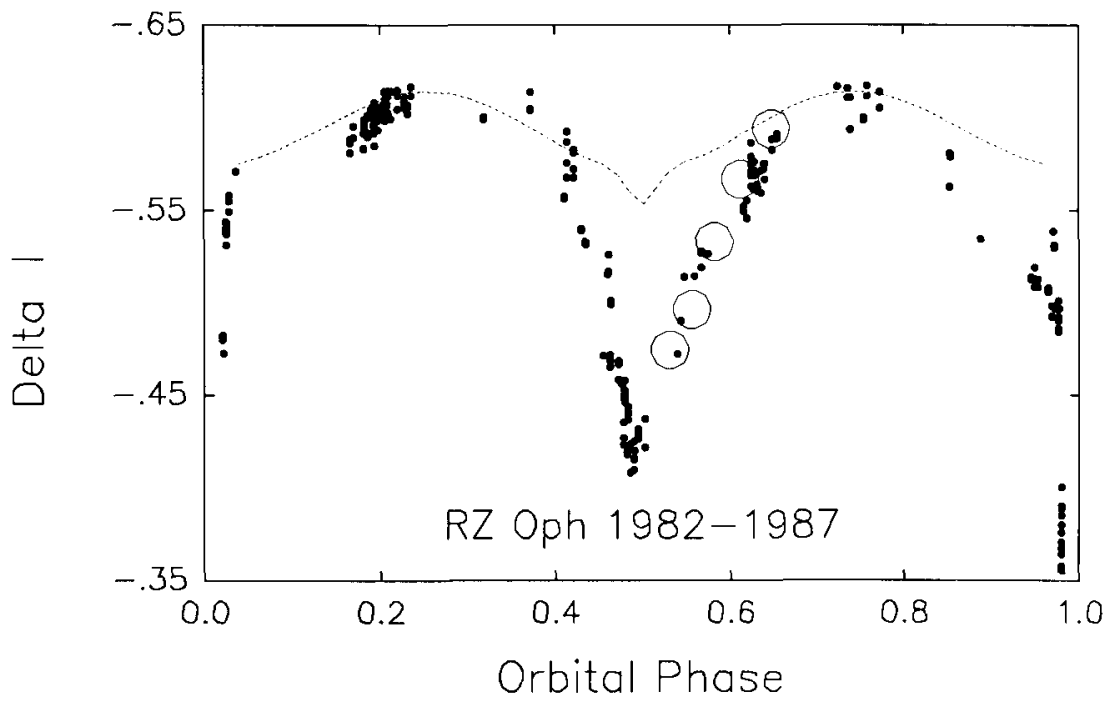

Fig. 5. Infrared observations of $\mathrm{RZ}$ Oph, mainly outside primary stellar eclipse. The dashed line is a predicted light curve, assuming only stellar light in the system. Open circles are predictions of a dusty disk model (see text). 


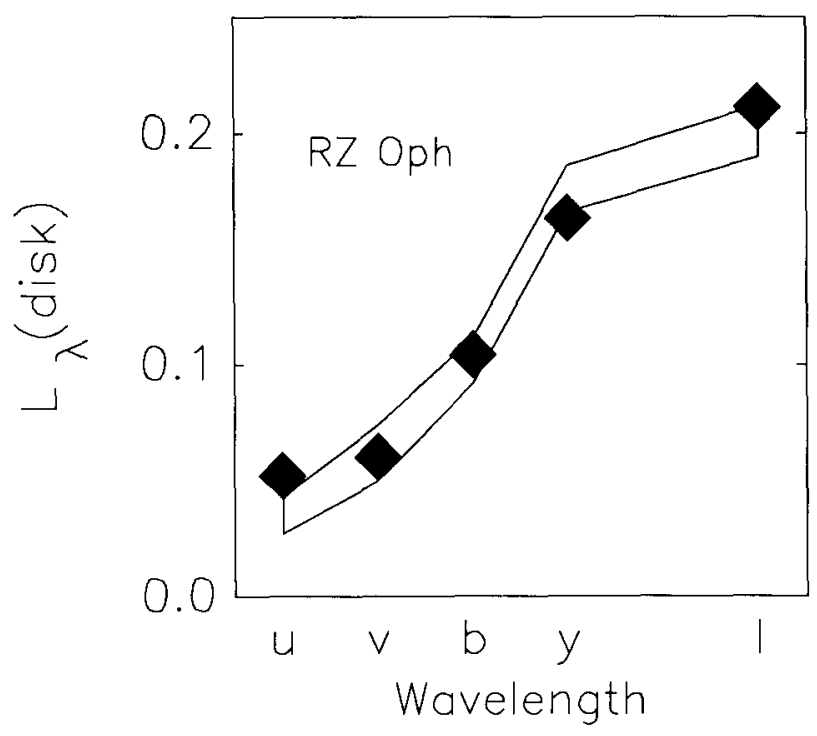

Fig. 6. Disk light in RZ Oph; diamonds are model predictions (see text).

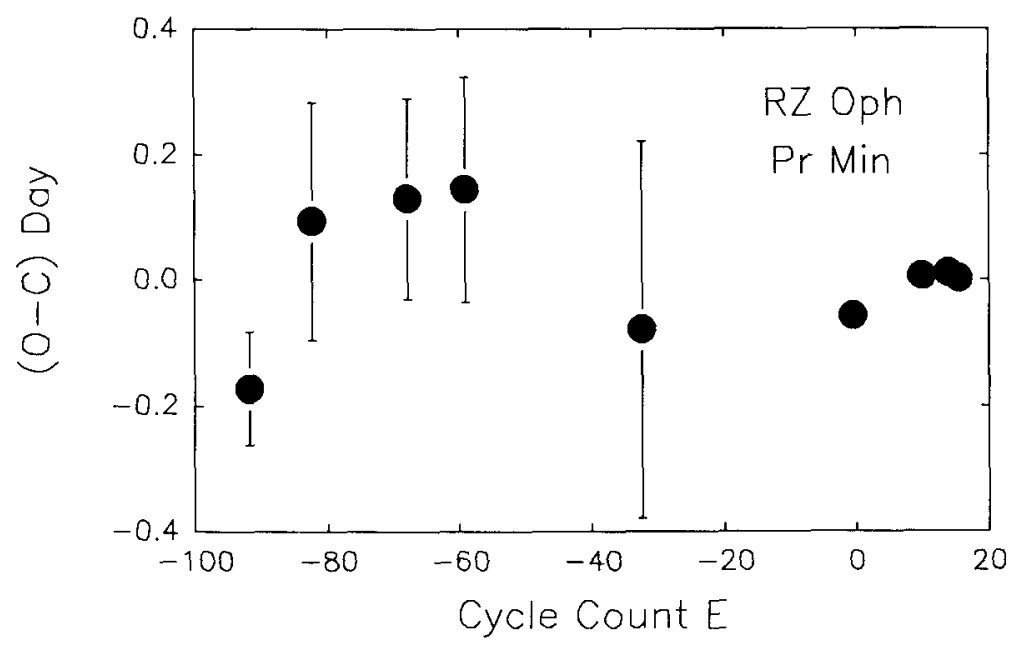

Fig. 7. Observed - calculated times of minima of RZ oph, from the new linear ephemeris. 


\section{DISCUSSION}

Rucinski emphasized the size of the dusty disks postulated for the systems of $\mathrm{KU}$ Cyg and RZ Oph. They would absorb much of the energy and might be expected to be moderately strong sources in the far infrared. Peters confirmed that $\mathrm{RZ} O \mathrm{ph}$ had indeed been detected by IRAS and the infrared flux was comparable to that found for $17 \mathrm{Lep}$ (B9Ve + M III, $P=260^{d}$ ), allowing for the very different visual apparent magnitudes. The two systems might be similar and seen at different inclinations.

Smak pointed out that obvious sources of asymmetry in these systems were the stream and the point at which it collides with the disk; obscuration by the stream could produce the asymmetries observed just before primary eclipse and just after secondary. 0lson replied that the wavelength dependence of the absorption did not correspond to that expected from a stream, as it did in, for example, U Sge (E.C. Olson Astr. J. 94, 1043, 1987). In reply to further questions from Smak, olson emphasized that his light-curve solutions by the Wilson-Devinney method had deliberately allowed only for the light of the two stars, in order to make the disk effects obvious. The disks appear at least to f111 the Roche lobes of the hotter star; that in RZ Oph is nearly an astronomical unt in radius.

Hilditch asked if the ratio of dust-to-gas derived by olson for the disks was comparable to those found in stellar winds from single latetype stars. Olson believed the latter not to be we11-known. In response to a query from Eaton about rate of mass-loss in the wind from the loser, 01son stated that the accretion rate in the disk must approach $10^{-6} \mathrm{~m}_{0} \mathrm{yr}^{-1}$, but the loss rate derived must depend on the unknown efficiency of the disk in capturing material. Eaton pointed out that UV observations of other long-period systems (e.g. 5 Cet and VV Cep) suggested that they contained similar structures (see also Eaton's remark after Plavec' talk, p.105) Livio emphasized the difficulty of forming large disks, such as that observed in RZ Oph, if the loser does not fill its Roche lobe; insufficient angular momentum would be accreted from an ordinary wind. He believed that either the loser fills the lobe precisely, or at least nearly enough to focus the wind into a stream resembling Roche-lobe overflow. Observers should re-examine the system.

Andersen reported an unpublished determination by $M$. Mayor of the rotational velocity of the secondary component which supported the view that the system is not semi-detached - unless the published mass-ratio is completely wrong. Scarfe remarked that new observations, with better coverage of the velocity-curve, confirmed the published mass-ratio. The velocity-curves correspond to circular orbits and show none of the distortions often associated with interaction of the two stars. olson conceded the angular-momentum problem pointed out by Livio and emphasized that KU Cyg is definitely semi-detached. Eggleton pointed out that if the hot star (gainer) accreted 20 per cent to 30 per cent of the mass lost by the loser, the total rate of mass-loss required is roughly consistent with the low mass of the cool star and the possible nuclear time-scale of a giant burning hellum in the core. (See also p.165) 